\title{
Principles of Rat Communication: Quantitative Parameters of Ultrasonic Calls in Rats
}

\author{
Stefan M. Brudzynski ${ }^{1, *}$
}

Ultrasonic calls used by rats during intraspecies communication have acoustic features, which can be selectively recognized by recipients, and possess a quantitative dimension reflecting the magnitude of the sender's response. This paper reviews basic principles of animal communication with particular attention to rat calls, and the features of ultrasonic calls, which could reflect such a quantitative aspect. Isolation calls of rat pups vary in frequency and duration and have changing sonographic structure over time. It is hypothesized that the quantitative "message" for the dam is encoded not only in the number of calls but also in frequency sweeps. The $22-\mathrm{kHz}$ alarm calls of adults are characterized by a relatively constant sound frequency, marginal frequency modulation, and remarkable variability in call duration. It is hypothesized that quantitative aspect of these calls may be encoded in call length. Finally, the $50-\mathrm{kHz}$ calls of adults, which are emitted in appetitive behavior are very short calls, with a relatively constant call duration, and a variable sound frequency. It is hypothesized that the peak frequency as well as the number of calls per time unit reflect the quantitative aspect in $50-\mathrm{kHz}$ calls.

KEY WORDS: Animal communication; isolation calls; rats; ultrasonic calls; rats; vocalization, $22-\mathrm{kHz}$ calls; $50-\mathrm{kHz}$ calls.

\section{INTRODUCTION}

Animal communication is a complex subject because of the enormous variability of ways that animals communicate. First, some basic definitions are presented from general biological perspectives to ensure unambiguous understanding and uniform interpretation of ultrasonic signals and signs contained in them. Second, the discussion will be narrowed to that of vocal communication in rats.

\section{Stimuli, Signals, and Signs}

All external stimuli arriving to an animal at any time are first subjected to a filtering process by specialized sensory and perceptual mechanisms, both at

\footnotetext{
${ }^{1}$ Department of Psychology and Centre for Neuroscience, Brock University, St. Catharines, Ontario, Canada.

* To whom correspondence should be addressed at Department of Psychology, Brock University, 500 Glenridge Avenue, St. Catharines, Ontario L2S 3Al Canada. Tel.: (905) 688-5550 ext. 4653. Fax: (905) 699-6922. e-mail: sbrudzyn@brocku.ca
}

peripheral and central levels. Thus, only a subset of all stimuli is perceived (Fig. 1). Any of these stimuli may potentially carry environmental information if interpreted appropriately. Those stimuli, animate or inanimate, which allow an animal to make predictions about the environment and thereby guide their behavior are termed biologically significant stimuli or cues (Hasson, 1994; Maynard-Smith and Harper, 2003). The subset of cues produced by animals and observed as a variety of behavioral manifestations can cause modification of the recipient's behavior, and therefore be used for communication. These animal-originating cues that alter the behavior of other organisms are termed signals (Maynert-Smith and Harper, 2003, see Fig. 1). Signal production evolved parallel to the receiver's response, i.e., the signal evolved because it was effective and it was effective because the receiver's response has also evolved (Maynert-Smith and Harper, 2003). This is also true for evolution of animal vocal communication.

The processes of central filtering, storing information, interpretation, prediction, and behavior 


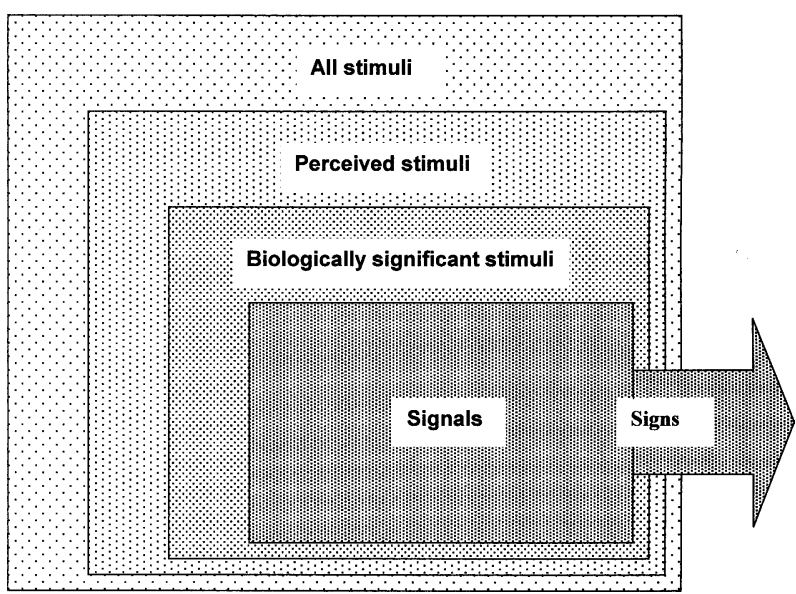

Fig. 1. Interrelationship among categories of stimuli and their importance for animal communication. Only a portion of all environmental stimuli is perceived and only a portion of those will be biologically significant (cues). Some of the cues become signals. Signals are used by animals for communication and contain signs (or "messages"), which are capable of influencing behavior of recipients. See text for further description of the terms.

initiation are each performed by specialized brain mechanisms at different levels of the central nervous system. Thus, the neural process associated with perception of an animal signal, its interpretation and the process of making decision about the response have to depend on some "mental" representation of the signalled quality in the brain of the receiver (Seyfarth and Cheney, 2003). The term "signal" contains two components: (1) a set of behvaioral manifestations, and (2) the quality being signalled or sign. The first component is perceived by the receiver but no action is taken, the second one, however, has a causal or guiding effect on receiver's behavior (Seyfarth and Cheney, 2003). Thus, from the definition, a true communicative signal is also a sign (Fig. 1). The signal is viewed as a sign only because it stands for its behavioral consequences. Thus, a sign is not a physical entity and it is also referred to as a sign function (Eco, 1976). The sign function of signals (in the first meaning) implies that the signal carries semiotic content, i.e., it stands for something else or signifies something of significance for recipients (counterpart of "meaning" or symbolic reference in the human language). Signals are, therefore, processed in the brain of receivers at least at two levels: sensory level and semiotic level. The concept of a sign is useful in ethological studies and will be explained further below. This terminology (stimulus, signal, and sign) was developed in semiotics - the study of sign systems, and in the general theory of communication codes (Eco, 1976; Danesi, 1993).

\section{A Definition of Animal Communication}

Animal communication includes the process of signal production, usually structured according to some code, signal transmission, and signal reception. Moreover, communication also includes behaviors elicited from the receiver, proving that the signal played its sign-function role. Thus, communication includes a number of processes associated with signal selection, coding, sending (producing), transmitting, receiving, decoding, and behavioral response of the receiver (for other theoretical details see Green and Marler, 1979).

The logic of linguistics is naturally anthropocentric (Morton, 1994). However, animal communication should not be studied in an anthropomorphic way. In contrast, we cannot know about an animal's subjective states. However, we can study animal communication if a number of conditions are fulfilled. Communication, and particularly acoustic communication, includes dynamic responses associated with sending and receiving coded signals as signs. Lack of a coded sign in a behavioral manifestation excludes it from being classified as communication. It is therefore necessary to assume that communicative signals carry semiotic content and, therefore, are capable of changing the behavior of the recipient. Even if the sign does not mean anything to the animal (in the human cognitive sense) the concept of the semiotic content of the signal is warranted if the signal initiates a neural process and causes predictable behavioral consequences in the recipient (Eco, 1976). Communication also occurs in a species-typical way and is a reproducible phenomenon. It is, however, subjected to changes during ontogeny. Taking into consideration an astonishing multitude of ways to communicate within many sensory modalities, this paper will be narrowed to rat's vocal communication in the ultrasonic range of sound frequencies.

\section{Semiotic versus Semantic Content in Rat Calls}

When referring to animal vocalizations, the term 'semantic content" ("meaning") should be replaced by the term 'semiotic content', which is understood more broadly as the symbolic or sign value of an acoustic signal (Danesi, 1993). Such a term has already been proposed for the entire 
discipline, which studies "messages" conveyed in animal communication, as zoosemiotics (Sebeok, $1963,1965)$. On the other hand, a recently proposed term, 'biosemiotics', for multitude of signs in signalling in all living systems seems to be too broad (Eder and Rembold, 1992). Semiotic content gradually evolved in animal communication system. Animal vocalizations originally evolved from semiotically arbitrary signals, which acquired symbolic reference (sign), and thus, communicative value (Marler, 1977). Ritualization was proposed as one of the evolutionary processes converting a cue into a signal (Meynert-Smith and Harper, 2003). This process also provides the basis for understanding the evolution of rats' ultrasonic communication, since biologically adaptive benefits resulted (for a full discussion see Nyby and Whitney, 1978). The sign (symbolic reference) in rat calls is usually related to a general situation, condition, or physiological state (motivational aspect), rather than to a specific object, feature, or function (referential aspect). Although, combination of both motivational and referential aspects in rat calls is possible (Marler et al., 1992). Thus, in addition to lacking the grammatical and syntactic complexity of human speech, rat communication also lacks the lexical precision of human communication. Nevertheless, rats can respond reliably to vocal signals of conspecifics and can discern the semiotic content even though, their calls may not be acoustically identical (Brudzynski and Chiu, 1995; Brudzynski, 2001; Brudzynski et al. 1999; Sales, 1991). This feature in animal's response is also observed for the vocalizations of other species and represents evidence for the existence of a recognizable sign function. Therefore, most animal vocalizations, including rat ultrasonic calls, are truly semiotic, i.e., containing a real sign communication system (for further discussion, see Busnel, 1977; Marler, 1977; Meynard-Smith and Harper, 2003). It is also true in relation to semiotic value of human vocalizations (laughter, grunting, crying, moaning, etc) or meaning of prosodic features of speech (e.g., change in pitch of voice), which are comparable homologues with the signals in animal communication (Nottebohm, 1975).

\section{Semiotic Sign Function}

Some vocal productions that are emitted accidently without relationship to the situation do not have any semiotic content (e.g., sneezing, or as a by-product of thoracic compression, Blumberg,
1992). These sounds remain without behavioral consequences and are not signals. On the other hand, a good example of a communicative signal is an infant call to a caregiver. Despite the genetically predetermined nature of these infant calls, maternal behavior and subsequent mutually initiated behavior provide evidence for vocal communication (Hofer, 1996; Shair et al., 2003). The simplest semiotic sign in this communication system might be the announcement of pup's presence and location and the maternal behavior confirms its proximity. This interpretation does not reflect a rat's "understanding" or "intentions", and is used for classification purposes and as a heuristic tool enabling evolutionary and sociobiological explanation of adaptations. This intrinsic, basic information about the presence of an individual is retained in vocal communication in all animals (Busnel, 1977).

The communicative value (sign) of rat calls may relate to several biological functions. They include: (a) a locating function (mentioned above) announcing presence of the emitter and enabling its localization, (b) an emotive function carrying information about the emitter's internal emotional valence, (c) a conative function mobilizing the recipient for action in a non-specific way, or activating its attention, (d) an alarming function informing about external danger (promoting for instance, freezing and other defensive responses), (e) agonistic function promoting escape, withdrawal or dispersion, (f) an affiliative function signalling approach and promoting conspecific contacts, and (g) a phatic function maintaining connections between individuals and maintaining cohesiveness of social groups in gregarious animals. This list does not exhaust all possibilities (e.g., alimentary or reproductive functions). Depending on the call's acoustic structure and the behavior elicited, a vocalization may be monosemic, i.e., play only one biological function and contain one sign (one meaning in human language), or may be polysemic by simultaneously providing several signs (multiple meanings in human language). Although, several acoustic parameters in rat calls may reflect monosemic function (Van der Poel and Miczek, 1991), it is likely that most rodent calls are polysemic.

It has been observed that animal communicatory signals may be complex and include complex displays or represent a set of multiple signals (Johnstone, 1996). These multiple signals, which could be studied using game theory models, may allow for more accurate assessment of a single sign 
by the receiver (so called 'backup signal' hypothesis), or they may represent a multiple sign communication ('multiple message' hypothesis) consistent with the polysemic function of the signals (Johnstone, 1996). For example, some features of $22 \mathrm{kHz}$ calls emitted as pairs of long and short calls (Brudzynski et al., 1993a) could represent such a multiple "message". Rat communication also seems probabilistic rather than deterministic. Further research is needed to better understand the multidimensional semiotic structure of rat communication.

\section{Communication of Rats by Means of Ultrasonic Calls}

In agreement with the definition of communication, the production of rat ultrasonic calls is an active and dynamic process, occurring in a species-typical, temporally defined way. Coordinated brainstem mechanisms are involved in the preparation of the respiratory system, larynx, and the articulatory apparatus for sound production with appropriate power of acoustic parameters (Jürgens, 2002). Rats first make an inspiration, the larynx is stabilized, and then vocal folds are tightly opposed leaving only a $1-2 \mathrm{~mm}$ opening (Roberts, 1975; Weisz et al., 2001). Forced expiration through the small opening produces a whistle-like call (Weisz, et al., 2001). For calls characterized by prolonged exhalations, a build-up of a significant abdominal pressure occurs, the expiratory air flow decreases, and the animal's head is lowered and protruded forward during the call (Brudzynski and Ociepa, 1992; Roberts, 1972). This response is centrally controlled, complex and integrated.

Ultrasonic calls are directed to other members of the species and are greatly influenced when a rat is isolated. In adult rats, calls are suppressed in the absence of other conspecifics (Blanchard et al., 1991; 1992; Brudzynski and Pniak, 2002) but potentiated in infants isolated from their mother (Hofer et al., 1998; Shair et al., 2003; Brunelli, 2005; D'Amato et al., 2005). The spontaneous appearance of alarm calls occurs in isolated adults but only after a prolonged, stressful isolation (Francis, 1977). In this situation, the biological function of these calls resembles that of infant isolation calls. Rat calls can be detected and perceived by other rats and they are reactogenic, i.e., capable of modifying the behavior of the recipients (Blanchard, et al., 1991; Brudzynski and Chiu, 1995; Sales, 1991; White and Barfield, 1989, 1990; White et al., 1993). For example, alarm calls induce behavior reflecting that the recipients can adequately make predictions about the environment (Blanchard et al., 1991). When the alarm calls were emitted by a dominant rat in response to a predator in an open area outside the burrow system, the entire colony responded. All members of the rat colony, including rats not present outside the burrow and who did not see the predator, hid for hours and responded with a long-lasting defensive behaviors, including vocal behavior, which further propagated the signal (Blanchard et al., 1991; Blanchard and Blanchard, 1989).

In summary, the emission of ultrasonic calls fulfills all the conditions of a true communication reflecting that these species-typical calls convey a sign for the receivers. However, the semiotic function (i.e., the "meaning", or "semantic content" of the acoustic signals in human terms) should not be interpreted from a subjective anthropocentric point of view. Rats lack human understanding and awareness.

\section{Quantitative Aspect of Ultrasonic Signals in Rats}

Ultrasonic calls in rats are emitted in situations, which guide the behavior of others with consequences for survival. The calls are well structured and have recognizable acoustic features (for example the frequency band of $20-35 \mathrm{kHz}$ and long call duration in alarm calls - Brudzynski and Chiu, 1995; Brudzynski, 2001; Kaltwasser, 1990). It is beneficial for survival to convey information reflecting the magnitude of the sign in the signal (in human terms, how strong is the meaning or how urgent is the message). It is known that the strength of the sign reflects the intensity of an emotional state in many mammalian communication systems (Brudzynski, 1981; Buchwald and Shipley, 1985; Jürgens, 1979; Scherer, 1985). The graded sign may also reflect the level of urgency in the response (Manser, 2001). This have important biological consequences because the stronger the sign, the higher the probability of responding and/or the shorter latency of the receiver response.

The magnitude of the sign must be coded in the signal, and thus, be reflected in the strength of the signal. Signal strength could be related to either the number of calls (of any type) per time unit, to the loudness of the calls, or other acoustic parameters. At present, there is not sufficient experimental data to make inference about loudness in rat calls. Consequently, other acoustic parameters of rat calls will be examined here. For example, call length might 
carry quantitative information. Such a relationship was demonstrated for growling in cats in response to a graded threat (Brudzynski et al., 1993b). The magnitude of pharmacologically induced vocalization, measured by a summed time of individual growls, was inversely and linearly proportional to the distance from the threat stimulus (i.e., the closer the threat the longer the calls). Also, moving threat stimuli caused longer vocalizations that stationary ones, and animals not familiar with the experimental situation vocalized more (i.e., with predominantly longer vocalizations) than those that had been exposed to that situation before (Brudzynski et al., 1993b). It is important to emphasize that the duration of individual calls, and not the number of vocalizations per time unit, was potentiated in response to the increasing levels of threat. The rate of calling only marginally contributed to the response augmentation (Brudzynski, 1981). Thus, this result suggests that the magnitude of the sign ("message") was coded in the duration of the individual vocalizations. Although, the number of vocalizations also could be used, to quantify the response, it is not the appropriate parameter in the cat.

Little information bears upon which acoustic parameter of the rat ultrasonic calls could carry the quantitative dimension of the sign apart from the rate of calling. In the following sections, three major types of rat ultrasonic calls will be analyzed with this goal in mind.

\section{Parameters of Rat Pup Isolation Calls}

Acoustic parameters, suitable for carrying sign strength should possess the highest degree of variability. Although, such parameters are obvious in adult rat calls, they are not so obvious in newborn rats. Pup ultrasonic calls are extremely variable in all acoustic parameters. Acoustic parameters in 10-17 day old Wistar pups differ by more than 65-70-times between minimal and maximal values (in single-call duration, sound-peak frequency, and bandwidth) (Brudzynski et al., 1999). Also, the call number and sonographic structure are equally variable (Elsner et al., 1990; Brudzynski et al., 1999). Pups appear not to utilize a single acoustic parameter coding the "message's" quantitative dimension but rather all parameters contribute to being found and attended to by the mother (locating function). Calls with abrupt beginnings and endings, and those that are short but variable in duration, are easier localized than long, fading calls.
It is also easier to localize frequency-modulated calls (sweeps) than calls with constant sound frequencies (Marler and Hamilton, 1966). All acoustic features facilitating sound localization are present in the pups' isolation calls, and this corroborates the critical importance of the dam's attention and attendance for the pups' survival. Although, increasing duration and peak frequency of the calls with age contribute to quantitative coding, (Brudzynski, et al., 1999), the number of frequency sweeps appears to be a main localization factor. Successive deep sweeps of sound frequency, analogous to an ambulance siren, are produced by a majority of pups. This pattern of calling requires a high-energy expenditure on the pup's part. Most pups produced calls with frequency sweeps and more than four times as many pups produced multiple sweeps rather than a single one (Fig. 2). Whether the magnitude, and number of sound-frequency sweeps is proportional to the urgency of the pup's need for maternal help is yet to be verified.

\section{Parameters of Adult 22-kHz Alarm Calls}

The 22-kHz alarm calls of adult rats are within a frequency range of $18-32 \mathrm{kHz}$ (Blanchard et al., 1992; Brudzynski and Ociepa, 1992; Brudzynski, 2001; Miczek et al., 1991; Nyby and Whitney, 1978; Sales, 1979; Van der Poel and Miczek, 1991), with a

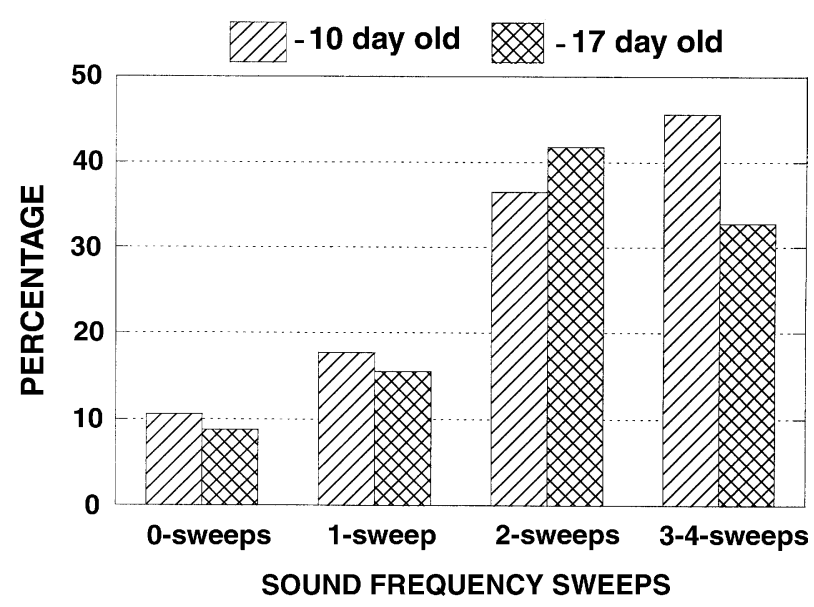

Fig. 2. Percentage distribution of selected categories of rat pup calls according to the number of sound frequency sweeps in their sonographic appearance for 10-day old pups (hatched bars) and for 17-day old pups (cross hatched bars). The total population of sonographically analyzed calls is $n=1211$ (78 pups) for 10-day old pups, and $n=438$ (71 pups) for 17-day old pups. The data originate from results contained in the study by Brudzynski, Kehoe and Callahan, (1999). 
narrow bandwidth of $1-6 \mathrm{kHz}$ and long call duration of approximately 300-3400 ms (Barfield and Geyer, 1975; Brudzynski et al., 1993; Brudzynski and Ociepa, 1992; Brudzynski, 2001; Miczek et al., 1991; Sales, 1979). These acoustic features are observed in behavioral situations associated with danger, threat, or distress. In particular, the calls with relatively constant sound frequency and long duration of single calls seem to be critical for the sign function of these calls (Brudzynski, 2001). In addition, artificially generated sounds with a similar constant frequency and long duration also had a powerful effect on rat behavior (Brudzynski and Chiu, 1995; Brudzynski, 2001; Sales, 1991).

The question arises as to how rats could express the degree of alarm (quantitative dimension). By far the biggest variability in this type of call is in duration (Fig. 3, Brudzynski et al., 1993a). The shortest and the longest calls differed by more than 195-times (20 ms versus $3940 \mathrm{~ms}$ ).

Consequently, I would suggest that this parameter is likely involved in the expression of the amount of alarm experienced. A direct test of the relationship between alarm calls duration and external danger magnitude has not yet been performed.

The defensive growling vocalizations of cats, where the duration of single calls is proportional to the magnitude of the external threat, can be induced by cholinergic stimulation of the brain (Brudzynski and Eckersdorf, 1988; Brudzynski et al., 1995).

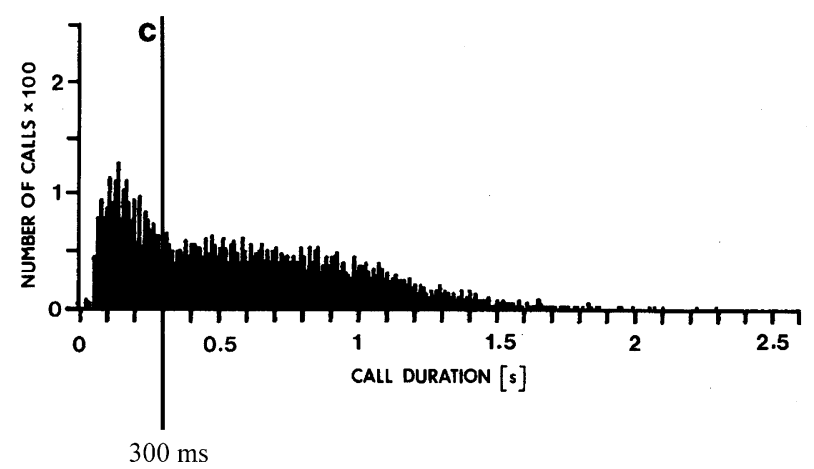

Fig. 3. Distribution of $22-\mathrm{kHz}$ calls of different call duration ( $n=6765$ calls) collected from 27 rats. The distribution was obtained from automated recordings and is corrected for accidental noises. The vertical line $(\mathrm{C})$ represents an arbitrary division for two subpopulations of short (less than $300 \mathrm{~ms}$ ) and long (more than $300 \mathrm{~ms}$ ) 22-kHz calls. Recent studies indicate that the number of calls shorter than $300 \mathrm{~ms}$ is smaller, relative to the longer calls, because of fragmentation of some of the long calls in the automated counting method. The figure (modified) is taken from Brudzynski et al., (1993a).
Similarly, ultrasonic alarm calls in rats can also be induced by direct cholinergic stimulation of homologue brain areas (Brudzynski, 1994). We hypothesized that the quantitative aspect of the rat response would be proportional to the dose of carbachol applied. However, analysis of call duration did not fully support this hypothesis (Brudzynski, 1994). Dose-dependent regulation of call length was observed only for the low end of the spectrum (calls as short as 100-300 ms). With higher doses, call duration actually decreased (Brudzynski, 1994). Rats emitted fewer long calls (more than $1000 \mathrm{~ms}$ in duration) and more short calls (shorter than $150-200 \mathrm{~ms})$. It is hypothesized that the $22-\mathrm{kHz}$ calls may not be a homogenous group of calls (Brudzynski et al., 1993a) or that endogenous acetylcholine, which initiates the response, may not code the quantitative aspect of the sign function.

\section{Parameters of Adult $50 \mathrm{kHz}$ Calls}

Short calls of the $50-\mathrm{kHz}$ type are emitted by juvenile and adult rats within a frequency range of 35-72 kHz (Blanchard, et al., 1993; Brudzynski and Pniak, 2002; Fu and Brudzynski, 1994; Kaltwasser, 1990; Takahashi et al, 1983; Wintink and Brudzynski, 2001;). The calls have short durations of $30-50 \mathrm{~ms}$ and a narrow bandwidth of $5-7 \mathrm{kHz}$. The results from several studies indicate that all of these parameters exhibit similar variability with the smallest and the biggest values of these parameters differing by 4.8 times for call duration, 4 times for sound frequency, and 4.6 times for bandwidth (Brudzynski and Pniak, 2002; Fu and Brudzynski, 1994; Wintink and Brudzynski, 2001). Pharmacological study with different doses of glutamate demonstrated, however, that unlike the other parameters, the peak sound frequency showed a significant positive correlation with glutamate dosage. Thus, peak sound frequency may be involved in coding the quantitative aspect of the sign function of $50-\mathrm{kHz}$ calls. It is, however, more probable that the quantitative sign dimension is simply coded in the number of calls emitted by rats in this particular type of calls. A dramatic increase in the number of $50-\mathrm{kHz}$ calls was observed in some social situations (Fig. 4) without any significant increase in mean peak frequency (Brudzynski and Pniak, 2002). The change from low to high levels of calling, which was generated in anticipation of a social contact, changed on average by 16 times. That the number of calls per time unit 


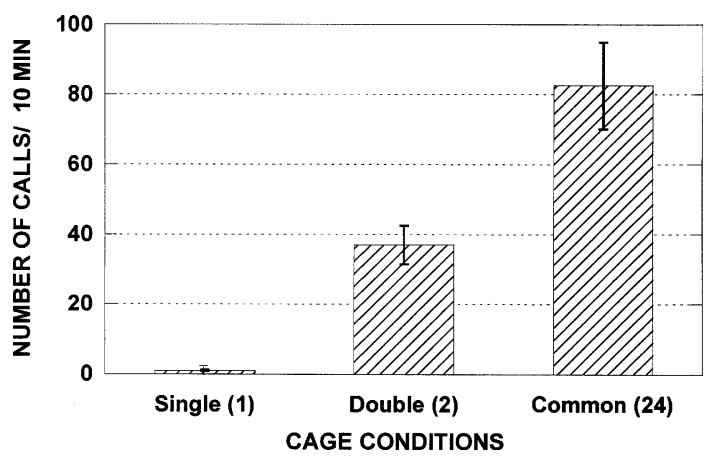

Fig. 4. Number of $50-\mathrm{kHz}$ calls $/ 10 \mathrm{~min}$ emitted spontaneously by adult rats in three behavioural caging conditions ( $n=24$ rats). Number of calls was recorded from a single rat in the cage. Calls were sonographically verified and counted. The recording were done in the cage occupied by one, and always the same rat [Single (1)], in the cage previously visited by another rat [Double (2)], and in the cage previously visited by 24 other rats [Common (24)]. There are significant differences in the number of calls among the conditions (ANOVA, F[2,23] $=15.3, p<0.001$ ). The acoustic parameters between conditions did not differ significantly and the maximal difference was less than $4.5 \%$ for any of the parameters. The figure (modified) is taken from Brudzynski and Pniak (2002).

expresses the important quantitative dimension is also very likely since call bandwidth is narrow and non-variable in this type of short call.

\section{Concluding Remarks}

Emission of ultrasonic calls in rats fulfills all conditions of a true intraspecific communication. The calls are structured signals, they convey a recognizable sign, and cause predictable behavioral consequences in the recipients. The magnitude of the sign may be encoded in the signal, which is an equivalent to the strength of the meaning or urgency of the message in human terms. There are several acoustic parameters of rat ultrasonic calls, which could code this quantitative sign aspect and they are likely to be represented by a physical parameter that shows high variability. It is postulated that the number of frequency sweeps in pup's separation call, duration of $22-\mathrm{kHz}$ calls, and the peak sound frequency and number of calls per time unit in $50-\mathrm{kHz}$ calls of adult rats may represent such highly variable quantitative parameters.

\section{ACKNOWLEDGMENTS}

This line of research is supported by the Natural Sciences and Engineering Research Council of Canada.

\section{REFERENCES}

Barfield, R. J., and Geyer, L. A. (1975). The ultrasonic postejaculatory vocalization and the postejaculatory refractory period of the male rat. J. Comp. Physiol. Psychol. 88:732-34.

Blanchard, R. J., and Blanchard, D. C. (1989). Antipredator defensive behaviors in a visible burrow system. J. Comp. Psychol. 103 (1):70-82.

Blanchard, R. J., Blanchard, D. C., Agullana, R., and Weiss, S. M. (1991). Twenty-two $\mathrm{kHz}$ alarm cries to presentation of a predator, by laboratory rats living in visible burrow systems. Physiol. Behav. 50:967-972.

Blanchard, R. J., Agullana, R. L., McGee, L., Weiss, S., and Blanchard, D. C. (1992). Sex differences in the incidence and sonographic characteristics of antipredator ultrasonic cries in the laboratory rat (Rattus norvegicus). J. Comp. Psychol. 106 (3):270-277.

Blanchard, R. J., Yudko, E. B., Blanchard, C. D., and Taukulis, H. K. (1993). High-frequency $(35-70 \mathrm{kHz})$ ultrasonic vocalization in rats confronted with anesthetized conspecific: Effects of gepirone, ethanol, and diazepam. Pharmacol. Biochem. Behav. 44:313-319.

Blumberg, M. S. (1992). Rodent ultrasonic short calls: locomotion, biomechanics, and communication. J. Comp. Psychol. 106:360-365.

Brudzynski, S. M. (1981). Growling component of vocalization as a quantitative index of carbachol-induced emotionaldefensive response in cats. Acta Neurobiol. 41:33-51.

Brudzynski, S. M. (1994). Ultrasonic vocalization induced by intracerebral carbachol in rats: localization and doseresponse study. Behav. Brain. Res. 63:133-143.

Brudzynski, S. M. (2001). Pharmacological and behavioral characteristics of $22 \mathrm{kHz}$ alarm calls in rats. Neurosci. Biobehav. Rev. 25:611-617.

Brudzynski, S. M., and Chiu, E. M. (1995). Behavioural responses of laboratory rats to playback of $22 \mathrm{kHz}$ ultrasonic calls. Physiol. Behav. 57:1039-1044.

Brudzynski, S. M., and Eckersdorf. (1988). Vocalization accompanying emotional-aversive response induced by carbachol in the cat. Reproducibility and dose-response study. Neuropsychopharmacology 1:311-320.

Brudzynski, S. M., and Ociepa, D. (1992). Ultrasonic vocalization of laboratory rats in response to handling and touch. Physiol. Behav. 52:655-660.

Brudzynski, S. M., and Pniak, A. (2002). Social contacts and production of $50 \mathrm{kHz}$ short ultrasonic calls in adult rats. J. Comp. Psychol. 116:73-82.

Brudzynski, S. M., Bihari, F., Ociepa, D., and Fu, X. W. (1993a). Analysis of $22 \mathrm{kHz}$ ultrasonic vocalization in laboratory rats: long and short calls. Physiol. Behav. 54:215-221.

Brudzynski, S. M., Eckersdorf, B., and Golebiewski, H. (1993b). Emotional-aversive nature of the behavioral response induced by carbachol in cats. J. Psychatr. Neurosci. 18:38-45.

Brudzynski, S. M., Eckersdorf, B., and Golebiewski, H. (1995). Regional specificity of the emotional-aversive response induced by carbachol in the cat brain: A quantitative mapping study. J Psychiatr Neurosci. 20:119-132.

Brudzynski, S. M., Kehoe, P., and Callahan, M. (1999). Sonographic structure of isolation-induced ultrasonic calls of rat pups. Dev. Psychobiol. 34:195-204.

Brunelli, S. A. (2005). Selective breeding for an infant phenotype: Rat pup ultrasonic vocalization (USV). Behav. Genet. 35:53-65.

Buchwald, J. S., and Shipley, C. A. (1985). Comparative model of infant cry. In B. M., Lester, and C. F. Z., Boukydis (eds.), Infant cry. New York:Plenum Press, pp. 279-305.

Busnel, R.-G. (1977). Acoustic communication. In T. S., Sebeok (ed.), How animals communicate. Bloomington: Indiana University Press, pp. 233-251. 
D'Amato, F. R., Scalera, E., Sarli, C., and Moles, A. (2005). Pup calls, mothers rush: Does maternal responsiveness affect the amount of ultrasonic vocalization in mouse pups? Behav Genet. 35:103-112.

Danesi, M. (1993). Messages and meanings: An introduction to semantics. Toronto: Canadian Scholars' Press Inc.

Eco, U. A. (1976). theory of semiotics. Bloomington: Indiana University Press.

Eder, J., and Rembold, H. (1992). Biosemiotics-a paradigm of biology. Biological signalling on the verge of deterministic chaos. Naturwissenschaften 79:60-67.

Elsner, J., Suter, D., and Alder, S. (1990). Microanalysis of ultrasound vocalizations of young rats: Assessment of the behavioural teratogenicity of methylmercury. Neurotoxicol Teratol. 12:7-14.

Francis, R. L. 22-kHz calls by isolated rats. Nature 265:236-238.

Fu, X. W., and Brudzynski, S. M. (1994). High-frequency ultrasonic vocalization induced by intracerebral glutamate in rats. Pharmacol. Biochem. Behav. 49:835-841.

Geen, S., and Marler, P. (1979). The analysis of animal communication. In P., Marler, and J. G., Vandenbergh (eds.), Handbook of behavioral neurobiology vol. 3. Social behavior and communication. New York:Plenum Press, pp 73-158.

Hasson, O. (1994). Cheating signals. J. Theor. Biol. 167:223-238.

Hofer, M. A. (1996). Multiple regulators of ultrasonic vocalization in the infant rat. Psychoneuroendocrinology 21:203-217.

Hofer, M. A., Masmela, J. R., Brunelli, S. A., and Shair, H. N. (1998). The ontogeny of maternal potentiation of the infant rats' isolation call. Dev. Psychobiol. 33:189-201.

Johnstone, R. A. (1996). Multiple displays in animal communication 'backup signals' and 'multiple messages'. Philos. Tr. R. S. S-B. 351:329-338.

Jürgens, U. (1979). Vocalization as emotional indicator. A neuroethological study in the squirrel monkey. Bhaviour 69:88117.

Jürgens, U. (2002). Neural pathways underlying vocal control. Neurosci. Biobehav. Rev. 26:235-258.

Kaltwasser, M.-Th. (1990). Acoustic signalling in the black rat (Rattus rattus). J. Comp. Psychol. 104:227-232.

Marler, P. (1977). The evolution of communication. In T. A., Sebeok (ed), How animals communicate. Bloomington: Indiana University Press, pp. 45-70.

Marler, P., Evans, C. S., and Hauser, M. D. (1992). Animal signals: motivational referential, or both? In H., Papoušek, U., Jürgens, and M., Papoušek (eds.), Nonverbal vocal communication Comparative and developmental approaches, Cambridge: Cambridge University Press, pp. 66-86.

Marler, P., and Hamilton, W. J. III. (1966). Mechanisms of animal behavior. New York: John Wiley \& Sons.

Manser, M. B. (2001). The acoustic structure of suricates' alarm calls varies with predator type and the level of response urgency. Proc. Roy. Soc. Lond-B. 268:2315-2324.

Maynard-Smith, J., and Harper, D. (2003). Animal signals. Oxford: Oxford University Press, p. 166.

Miczek, K. A., Tornatzky, W., and Vivian, J. (1991). Ethology and Neuropharmacology: Rodent ultrasounds. In Olivier, B., J., Mos, and J., Slangen (eds.), Animal Models in Psychopharmacology, Boston: Birkhäuser Verlag, pp. 409-427.
Morton, E. S. (1944). Sound symbolism and its role in nonhuman vertebrate communication. In L., Hinton, J., Nichols, and J. J., Ohala (eds.), Sound Symbolism, Cambridge England: Cambridge Imoversotu Press, pp. 348-365.

Nottebohm, F. (1975). A zoologist's view of some language phenomena with particular emphasis on vocal learning. In E. H., Lennenberg, and E., Lennenberg (eds.), Foundations of language development vol. 1, New York: Academic Press, pp. 61-103.

Nyby, J., and Whitney, G. (1978). Ultrasonic communication of adult myomorph rodents. Neurosci. Biobehav. Rev. 2:1-14.

Roberts, L. H. (1972). Correlation of respiration and ultrasound production in rodents and bats. J. Zool. Lond. 168:439-449.

Roberts, L. H. (1975). The rodent ultrasound production mechanism. Ultrasonics 13:83-88.

Sales, G. D. (1979). Strain differences in the ultrasonic behavior of rats (Rattus norvegicus). Am. Zool. 19:513-29.

Sales, G. D. (1991). The effect of $22 \mathrm{kHz}$ calls and artificial $38 \mathrm{kHz}$ signals on activity in rats. Behav. Processes. 24:83-93.

Scherer, K. R. (1985). Vocal affect signalling: A comparative approach. In J. S., Rosenblatt, C., Beer, M.-C., Busnel, and P. J. B., Slater (eds.), Advances in study of behavior vol. 15, Orlando: Academic Press Inc, pp. 189-244.

Sebeok, ThA. (1963). Communication in animals and men. Language 39:448-466.

Sebeok, ThA. (1965). Zoosemiotics: Juncture of semiotics and the biological study of behavior. Science 147 (3657):492-493.

Seyfarth, R. M., and Cheney, D. L. (2003). Signallers and receivers in animal communication. Ann. Rev. Psychol. 54:145173.

Shair, H. N., Brunelli, S. A., Masmela, J. R., Boone, E., and Hofer, M. A. (2003). Social, thermal, and temporal influences on isolation-induced and maternally potentiated ultrasonic vocalizations of rat pups. Dev. Psychobiol. 42:206-222.

Takahashi, L. K., Thomas, D. A., and Barfield, R. J. (1983). Analysis of ultrasonic vocalizations emitted by residents during aggressive encounters among rats (Rattus norvegicus). J. Comp. Psychol. 97:207-212.

Van der Poel, A. M., and Miczek, K. A. (1991). Long ultrasonic calls in male rats following mating, defeat and aversive stimulation: frequency modulation and bout structure. Behavior 119:127-142.

Weisz, D. J., Yang, B. Y., Fung, K., and Amirali, A. (2001). The mechanism of ultrasonic vocalization in the rat. Soc. Neurosci. Abstr. 27:\#88.19.

White, N. R., and Barfield, R. J. (1989). Playback of female rat ultrasonic vocalizations during sexual behavior. Physiol. Behav. 45:229-233.

Wintink, A. J., and Brudzynski, S. M. (2001). The related roles of dopamine and glutamate in the initiation of $50-\mathrm{kHz}$ ultrasonic calls in adult rats. Pharmacol. Biochem. Behav. 70:317323.

White, N. R., and Barfield, R. J. (1990). Effects of male pre-ejaculatory vocalizations on female receptive behavior in the rat (Rattus norvegicus). J. Comp. Psychol. 104:140-146.

White, N. R., Gonzales, R. N., and Barfield, R. J. (1993). Do vocalizations of the male rat elicit calling from the female? Behav. Neurol. Biol. 59:76-78. 